\title{
Multi-resolution LOD Volume Rendering in Medicine
}

\author{
Kai Xie ${ }^{\mathrm{a}, *}$, Jie Yang ${ }^{\mathrm{a}}$, and Yue Min Zhu ${ }^{\mathrm{b}}$ \\ ${ }^{a}$ Inst. of Image Processing \& Pattern Recognition, \\ Shanghai Jiaotong Univ., 200030 Shanghai,China \\ ${ }^{\mathrm{b}}$ CREATIS - CNRS research unit 5515 \& INSERM unit 630, \\ 69621 Villeurbanne, France \\ xie_kai2001@sjtu.edu.cn
}

\begin{abstract}
This paper presents a level of detail (LOD) selection algorithm for multi-resolution volume rendering using $3 \mathrm{D}$ texture mapping. It uses an adaptive scheme that renders the volume in a region-of-interest at a high resolution and the volume away from this region at progressively lower resolutions. The algorithm is based on several important criteria, rendering is done adaptively by selecting high-resolution cells close to a center of attention and low-resolution cells away from this area. In addition, our hierarchical levelof-detail representation guarantees consistent interpolation between different resolution levels. Experiments have been applied to a number of large medical data and produced high quality images at interactive frame rates using standard PC hardware.
\end{abstract}

\section{Introduction}

Volume rendering of large data sets is a very common task in many areas in medicine. To address the challenge, researchers have proposed various algorithms. Among the existing techniques, hierarchical rendering algorithms can effectively control the tradeoff between quality and speed, and thus show a great potential. In essence, hierarchical methods first create a multi-resolution representation for the volume. Data of different resolutions in different regions are chosen for rendering. The effectiveness of hierarchical algorithms relies on their ability to adaptively simplify rendering in regions where data are unimportant or uninteresting, so that both the memory and computational cost can be reduced without significantly affecting the rendering quality.

Hierarchical volume rendering algorithms [1][2][3] can accelerate the speed of rendering. The selection of appropriate volume resolutions, or levels of detail (LOD), is often done for the different applications. Although various LOD selection algorithms for polygon rendering systems [5][6] are available, LOD selection algorithms for volume rendering, however, are still scarce. Frequently used approaches such as selecting the volume resolutions based on user specified error tolerances [4], based on the volume block's distance to the viewpoint and the angle to the view vector [1][2] are difficult to guarantee the rendering quality and the continuity at level boundaries while reducing the rendering time. 
This paper presents a LOD selection algorithm for rendering hierarchical volumes using 3D texture hardware. The main focus of our algorithm is adaptive LOD selection based on several important criteria: Maximum opacity, Distance to the view point, Projection area and Gaze distance. In addition, we reduce the run-time performance of volume rendering and insure interpolation consistency between levels.

The rest of the paper is organized as follows. In section 2, we present our multiresolution rendering algorithm in detail. Experimental results are discussed in section 3. Conclusions are discussed in section 4.

\section{Multi-resolution LOD Volume Rendering}

We develop a multi-resolution hierarchy that allows consistent interpolation between levels and give a solution to the rendering artifacts that still persist when rendering two differing but adjacent levels. In addition, to make sure that regions of interest are rendered at a higher quality, our algorithm allocates the different hierarchy to different subvolumes based on several important criteria, which can be simple heuristics such as the distance to the view point, the volume opacity, or other application-specific criteria. In the following, we describe our algorithm in detail.

\subsection{The Multi-resolution Texture Hierarchy}

Prior to the rendering, the volume data set is subdivided into multiple subvolumes of smaller size, which get assigned the chunk of texture that is necessary to render the subvolume at the original resolution. Each subvolume builds its own local hierarchy by constructing copies of the original texture at ever coarser resolution. These different levels-of-detail are stored in additional texture maps as shown in Figure 1. On every level the size of texture elements increases by a factor of two. Note that at subvolume boundaries on the same level, texture elements have to be included in multiple subvolumes in order to guarantee continuous texture interpolation (filled areas).

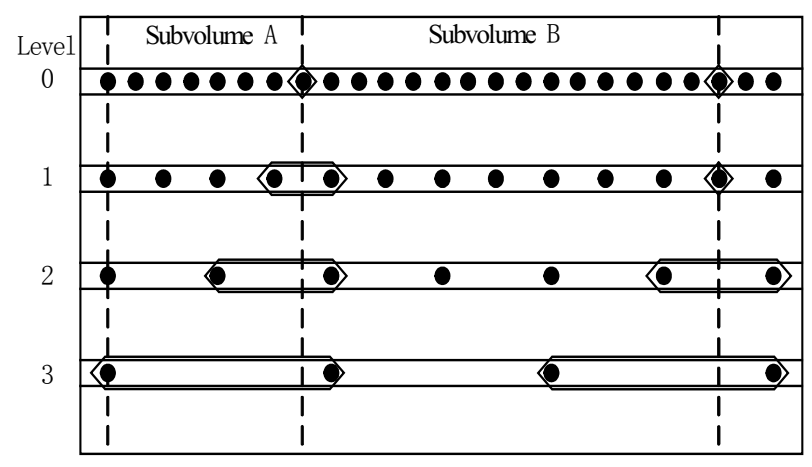

Fig. 1. One-dimensional of a data set with four levels of detail

The original data set is represented by textures of level 0 . The width of texture elements on level $k$ is twice the width of elements on level $k$ - 1 , whereas the texture 
size is reduced by a factor of two. Texture elements on different resolution levels are aligned in such a way, that their centers on a certain level correspond to the center of an even element on the next finer level. Since the geometry or shape of bricks will be retained throughout the hierarchy, the domain of the underlying texture function, necessary to compute appropriate texture coordinates, has to be adapted accordingly.

On the first level, the domain of texture coordinates ranges from the center of the first element to the center of the last one. Due to the alignment of voxels on different levels, borders of the texture function domain on coarser levels often fall between two adjacent voxels. In this case additional voxels are needed in order to guarantee correct interpolation. On the other hand, since the width of texture elements on each level is known and because the shape of subvolumes is not going to be modified, offsets for correct calculation of texture coordinates can always be determined.

We should note that it is always necessary to expand textures in order to cope with the power-of-two restriction imposed by the OpenGL implementation. However, a hierarchical technique will be outlined below that allows us to effectively avoid unnecessary texture elements.

\subsection{Continuous Level Transitions}

If local texture hierarchies are constructed as described, interpolation artifacts at the boundaries between adjacent bricks at the same level do not appear. This is because boundary voxels are shared by adjacent subvolumes. However, this does not apply to adjacent bricks rendered on different levels.

Therefore, it is necessary to slightly modify the treatment of level transitions to meet the continuity requirements: in the level-of-detail representation the continuity at level transitions can be established by letting the finer cells to the left and to the right of the subvolume boundary interpolate the scalar field from the coarser level (Figure 2). For cells with even index in each dimension this is equivalent to a copy operation as they correspond exactly to a cell on the next coarser level. This procedure only adapts the subvolume textures on the finer level. Thus, with the combination of the proposed multi-resolution representation together with appropriately re-sampled values at level transitions we guarantee the continuity of the $3 \mathrm{D}$ scalar field.

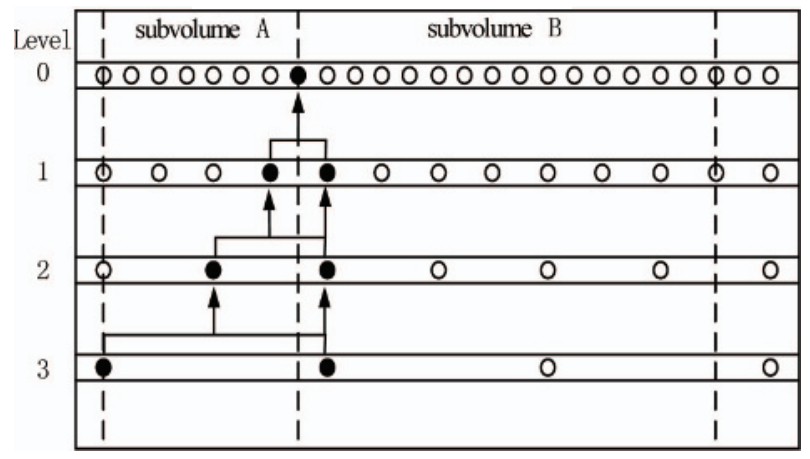

Fig. 2. Adaption of adjacent subvolumes with different level-of-detail to ensure consistent texture interpolation 
Note in particular, that we restrict transitions to differ by at most one level in order to maintain the continuity between levels. However, this does not impose a real restriction as higher transitions can be achieved by consecutive transitions of one level.

The number of voxels that has to be adapted depends on the position of the brick boundary relative to the voxel coordinates. Either the boundary is located at the center of one voxel, as is the case on level 0, or between two voxels, as is the case on other levels. In the first case only one voxel needs to be adapted while both voxels have to be modified in the latter case. Due to the overlap between bricks, adaption has to be performed whenever a subvolume is adjacent to a coarser one.

\subsection{Level-Wise Texture Merging}

The enlargement of textures in order to guarantee continuous level transitions leads to significant overhead in the texture memory that is used. Refer to the example shown in Figure 2. If we want to render the information represented by the first 31 voxels on level 0 , we need two textures of size 16 for the finest level and two textures of size 16 on level 1 whereas only nine texture elements are necessary to store the information. Effectively there is no saving of texture memory when switching from level 0 to level 1. Considering higher levels leads to similar results, as only half of the voxels of every texture - plus one or two for overlap - contain non-redundant information.

This overhead can be minimized by merging the texture data of adjacent subvolumes into a single texture as shown in Figure 3. This figure demonstrates the one-dimensional analogue. For each level-of-detail the same texture size is used. In level 0 each brick has a texture of its own. As in any direction only half the voxels of a particular level are needed on the next coarser level, eight adjacent subvolumes can be represented by the same texture map, with appropriate texture coordinates. On higher levels, the number of subvolumes sharing the same texture map is multiplied by a factor of 8 , which finally results in an octree-like hierarchy of texture maps.

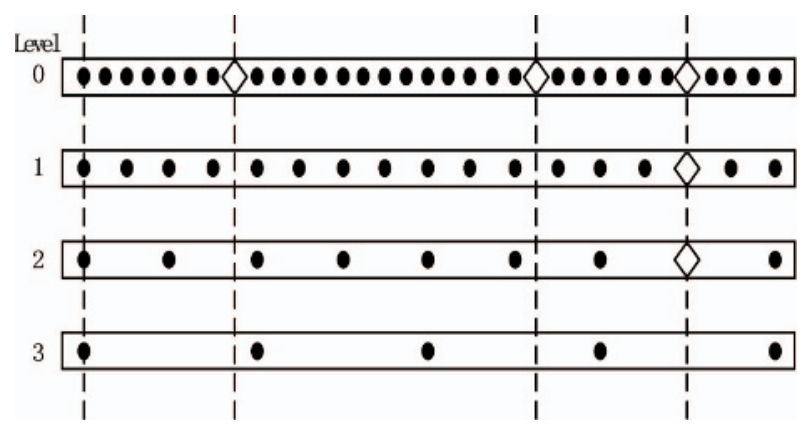

Fig. 3. The hierarchy of storing the textures of subvolumes

Merging textures works best when the starting number of subvolumes in every direction matches a power of two. If more than $2^{1-1}$ bricks are created by the subdivision of the initial data set, where 1 is the number of levels used for rendering, additional subvolumes will be generated that build their own set of textures as 
described in subsection 3.1. If fewer subvolumes are generated, then the depth of the texture hierarchy has to be reduced by storing textures on the coarsest level individually. We utilize a texture manager object for administration of the texture hierarchy. This object creates the desired texture maps and assigns appropriate subtextures to the subvolumes requesting texture memory.

\subsection{Opacity Correction}

When rendering subvolumes at different levels of hierarchy, the opacity properties of the subvolumes are different. The classical rendering algorithms depend on using the same sampling along rays for each pixel. But in the context of a multi-resolution format, the volume is sampled in different ways, and at varying resolutions. To preserve optical properties between tiles of different resolutions, we must modify the transfer functions for those subvolumes generated by subsampling the original texture.
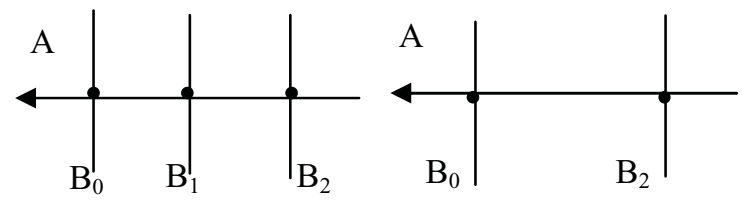

Fig. 4. Sampling a texture at two different resolutions

Figure 4 shows an example where we have sampled a texture at two different resolutions - one is half the resolution of the other. Each sample $B_{i}$ has an associated color $c_{i}$ and an opacity value $\alpha_{i}$. By considering only the first three samples, the resulting colors $A$ and $A^{\prime}$ are given by

$$
\begin{gathered}
A=\alpha_{0} c_{0}+\left(1-\alpha_{0}\right) \alpha_{1} c_{1}+\left(1-\alpha_{0}\right)\left(1-\alpha_{1}\right) C_{2} \\
A^{\prime}=\alpha_{0}^{\prime} c_{0}+\left(1-\alpha_{0}^{\prime}\right) C_{2}^{\prime}
\end{gathered}
$$

Where $C_{2}$ is the incoming color from samples $B_{2}, B_{3} \cdots, C_{2}^{\prime}$ is the color calculated as a result of the samples $B_{4}, B_{6} \ldots$

However, if we compute the total-accumulated opacities $D$ and $D^{\prime}$, we obtain

$$
\begin{gathered}
D=\alpha_{0}+\left(1-\alpha_{0}\right) \alpha_{1}+\left(1-\alpha_{0}\right)\left(1-\alpha_{1}\right) D_{2} \\
D^{\prime}=\alpha_{0}^{\prime}+\left(1-\alpha_{0}^{\prime}\right) D_{2}^{\prime}
\end{gathered}
$$


Assuming that the accumulated opacities are equal at the even samples, it follows that $D_{2}=D_{2}^{\prime}$ and $D=D^{\prime}$, i.e.,

$$
\alpha_{0}+\left(1-\alpha_{0}\right) \alpha_{1}+\left(1-\alpha_{0}\right)\left(1-\alpha_{1}\right) D_{2}=\alpha_{0}^{\prime}+\left(1-\alpha_{0}^{\prime}\right) D_{2}^{\prime}
$$

Solving this equation for $\alpha_{0}^{\prime}$, one obtains

$$
\alpha_{0}^{\prime}=1-\left(1-\alpha_{0}\right)\left(1-\alpha_{1}\right)
$$

By assuming that $\alpha_{1}=\alpha_{0}+\mathcal{E}$ (where $\varepsilon$ is a very small number), we obtain the equation

$$
\alpha_{0}^{\prime}=1-\left(1-\alpha_{0}\right)^{2}+O(\varepsilon)
$$

Therefore, we modify the transfer function of the parent (coarser) texture by

$$
\alpha^{\prime}=1-\left(1-\alpha_{0}\right)^{2}
$$

for all opacity values in the subsampled texture to minimize the artifacts between subvolumes. This formula is used when applying the transfer function to a level of the texture hierarchy.

\subsection{Adaptive Level-of-Detail}

The initial size of each subvolume has to be specified in advance before the multivolume representation is constructed. Multi-criteria are applied to LOD selection .

- Maximum opacity: The maximum opacity of a subvolume is determined by the highest opacity of all the voxels in the subvolume. The rationale behind is that a more opaque region in the volume should be rendered in a higher accuracy.

- Distance to the view point: Here the distance is calculated from the center of the subvolume to the view point. For those subvolumes that are closer to the view point, as they have high importance value, they will be rendered in a high quality. For other subvolumes, as they are farther away and may be occluded, will be rendered in a low quality.

- Projection area: Projection area is calculated based on the bounding box of the subvolume. For those subvolumes with high projection area, a higher quality rendering should be assigned to them.

- Gaze distance: This parameter is especially useful for gaze-directed rendering. Gaze distance is the distance between the center of the gaze area and the center of the projected area of a subvolume. For those regions that are closer to the gaze area, we ensure a higher image quality. For those regions that are farther away from the gaze area, the image becomes blur because lower resolution data are used.

Furthermore, as long as no level transitions greater than one are specified, our approach allows us to use arbitrary texture resolution for every subvolume. 


\subsection{Temporal Coherence Consideration}

The above LOD selection procedure will be executed at every frame, which could cause the subvolume's LOD to change frequently. However, frequent changes of the volume LODs can cause flickering when the user changes views. To solve this problem, we take special care to maintain the temporal coherence in consecutive rendering frames. We realize temporal coherence by recording the importance value of each subvolume from the last rendering. When a new frame is being rendered, if the user changes the viewing direction or gaze area, the importance value of each subvolume will be recalculated. For those subvolumes whose importance values do not differ too much (we use a threshold of percentage to decide this), we do not change its LOD in the new frame.

\section{Results and Discussion}

We have implemented our LOD volume rendering algorithm on a Windows 2000 PC with a $1.7 \mathrm{GHz}$ AMD processor and NVIDIA GeForceFx graphics accelerator with $128 \mathrm{MB}$ of Video RAM. Three data sets were used for the experiments.

Table 1. Rendering speed for the various data sets

\begin{tabular}{|c|c|c|c|c|c|}
\hline \multirow{2}{*}{ Model } & \multirow{2}{*}{ Data Set Size } & \multirow{2}{*}{$\begin{array}{l}\text { Texture } \\
\text { memory }\end{array}$} & \multicolumn{3}{|c|}{ Rendering Speed(fps) } \\
\hline & & & our algorithm & ray casting & shear-warp \\
\hline \multirow{3}{*}{ MRIBrain } & \multirow{3}{*}{$256^{2} \times 109$} & $100 \%$ & 17.8 & 6.4 & 21.5 \\
\hline & & $54 \%$ & 21.4 & - & - \\
\hline & & $33 \%$ & 24.2 & - & - \\
\hline \multirow{3}{*}{ Foot } & \multirow{3}{*}{$102 \times 247 \times 200$} & $100 \%$ & 18.6 & 5.7 & 20.1 \\
\hline & & $53 \%$ & 22.5 & - & - \\
\hline & & $27 \%$ & 27.4 & & \\
\hline \multirow{3}{*}{ Spine } & \multirow{3}{*}{$256^{2} \times 299$} & $100 \%$ & 14.3 & 5.2 & 18.4 \\
\hline & & $57 \%$ & 16.8 & - & - \\
\hline & & $29 \%$ & 19.1 & - & - \\
\hline
\end{tabular}

Abbreviations: fps $=$ frames per second.

In Figure 5, results of the proposed level-of-detail rendering technique applied to MRI-Brain of size $2562 \times 109$ are shown. In the leftmost image, all subvolumes were rendered with full resolution. In the middle image only $54 \%$ of the original texture memory was used, while on the right, only $33 \%$ was used (Table 1). The number of texture lookups was reduced, from $59 \%$ to $35 \%$. This leads to improved rendering performance. Whereas the frame rate is $17.8 \mathrm{frames} / \mathrm{s}$ with the full resolution data set, the level-of-detail representation used for the rightmost image allows us to create a frame rate is 24.2 frames/s (Table 1). The rendering speed of our algorithm is quick compared to other volume rendering techniques, such as ray casting and shear-warp. 
In Figure 6, we use gaze-directed rendering to show the results when the gaze area is placed at different parts of the viewport.

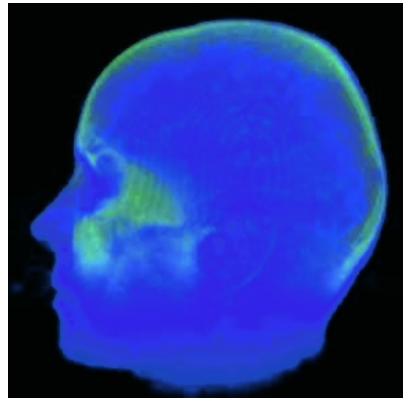

(a)

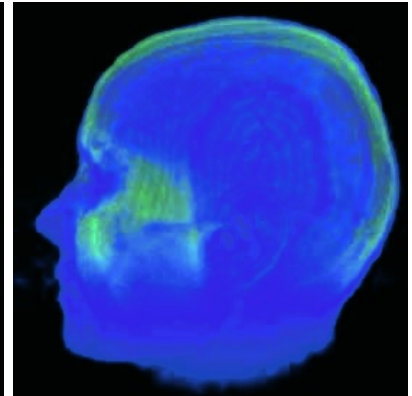

(b)

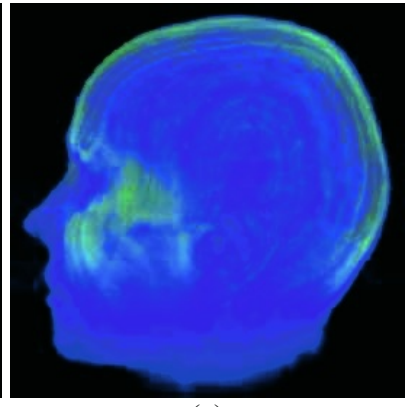

(c)

Fig. 5. On the left, the data set is displayed with full resolution.In the middle, two different levels of detail are used with lower resolution in the back. This reduces texture memory consumption to $54 \%$. On the right, the adaptive representation using four levels of detail from front-to-back requires only $33 \%$ of the original texture memory

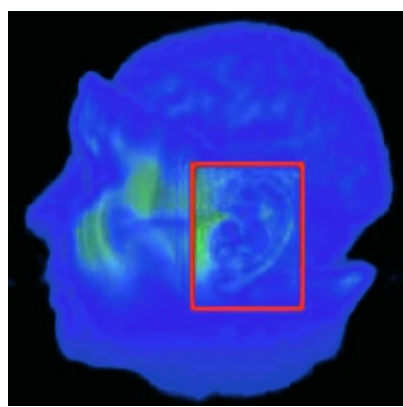

(a)

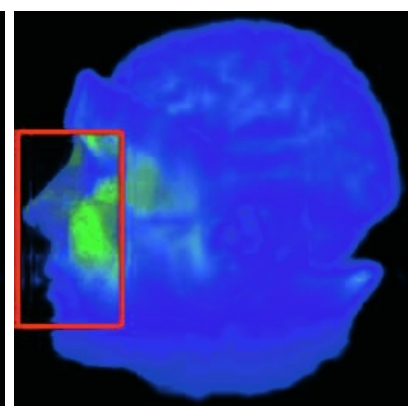

(b)

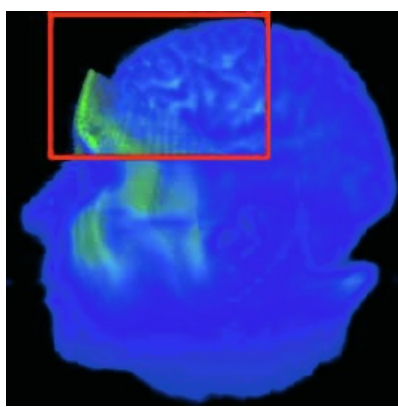

(c)

Fig. 6. Gaze-directed rendering. (a)(b)(c) show the results when the gaze area is placed at different parts of the viewport

\section{Conclusions}

In this work we have emphasized a multi-resolution approach for the rendering of large-scale volume data via 3D textures. The major contribution here is that we entirely avoid artifacts that occur due to incorrect texture interpolation and opacity correction at subvolume boundaries. In this respect, we have developed two beneficial extensions that guarantee continuous transitions between different levels of detail, and yield correct pixel opacities. 


\section{References}

1. E. LaMar, B. Hamann, and K. Joy. Multiresolution techniques for interactive texture-based volume visualization. In Proceedings of Visualization '99, pages 355-361. IEEE Computer Society Press, Los Alamitos, CA,1999.

2. M. Weiler, R. Westermann, C. Hansen, K. Zimmerman,and T. Ertl. Level-of-detail volume rendering via $3 \mathrm{~d}$ textures. In Proceedings of 2000 Symposium on Volume Visualization, pages 7-13. ACM SIGGRAPH, 2000.

3. D. Ellsworth, L. Chiang, and H.-W. Shen. Accelerating time-varying hardware volume rendering using tsp trees and color-based error metrics. In Proceedings of 2000 Symposium on Volume Visualization. ACM SIGGRAPH,2000.

4. D. Laur and P. Hanrahan. Hierarchical splating: A progressive refinement algorithm for volume rendering. In Proceedings of SIGGRAPH 91, pages 285-287. ACM SIGGRAPH, 1991.

5. T. Funkhouser and C. Sequin. Adaptive display algorithms for interactive frame rate during visualization of virtual environment. In Proceedings of SIGGRAPH 93, pages 247-254. ACM SIGGRAPH, 1993.

6. E. Gobbetti and E. Bouvier. Time-critical multiresolution scene rendering. In Proceedings of 1999 Symposium on Volume Visualization, pages 123-130. ACM SIGGRAPH, 1999. 